PROCEEDINGS OF THE

AMERICAN MATHEMATICAL SOCIETY

Volume 125, Number 9, September 1997, Pages 2661-2670

S 0002-9939(97)03885-9

\title{
ON THE IDEAL-TRIANGULARIZABILITY OF POSITIVE OPERATORS ON BANACH LATTICES
}

\author{
MOHAMMED TAGHI JAHANDIDEH
}

(Communicated by Palle E. T. Jorgensen)

\begin{abstract}
There are some known results that guarantee the existence of a nontrivial closed invariant ideal for a quasinilpotent positive operator on an $A M$-space with unit or a Banach lattice whose positive cone contains an extreme ray. Some recent results also guarantee the existence of such ideals for certain positive operators, e.g. a compact quasinilpotent positive operator, on an arbitrary Banach lattice. The main object of this article is to use these results in constructing a maximal closed ideal chain, each of whose members is invariant under a certain collection of operators that are related to compact positive operators, or to quasinilpotent positive operators.
\end{abstract}

\section{Preliminary definitions \& Lemmas}

Throughout the article we will employ the terminology of the books [3] and [10] and assume familiarity with basic definitions and results of these books. We shall also adhere to the following conventions and definitions.

Unless otherwise stated $\mathbf{N}$ is the set of all natural numbers, $X$ is a general real or complex Banach space, $\mathcal{B}(X)$ is the collection of all bounded linear operators on $X, E$ is a real Banach lattice, $E_{+}=\{x \in E: x \geq 0\}$ is the positive cone of $E$, and the topology on $\mathcal{B}(X)$ is the norm topology.

By a closed invariant ideal we mean a closed ideal that is taken into itself by the given operator. A subset $\Gamma$ of $\mathcal{B}(E)$ is called decomposable if there exists a nontrivial closed ideal that is invariant for all operators in $\Gamma$, otherwise $\Gamma$ is called indecomposable. The collection of all closed invariant ideals (subspaces) of a subset $\Gamma$ of $\mathcal{B}(E)(\mathcal{B}(X))$ is denoted by $\operatorname{Ilat}(\Gamma)(\operatorname{Lat}(\Gamma))$. If $\Gamma=\{T\}$, where $T \in \mathcal{B}(X)$, we simply use $T$ instead of $\Gamma$.

A closed ideal of $E$ is p-hyperinvariant for a positive operator $T$ on $E$ if it is invariant under every positive operator $S$ on $E$ which commutes with $T$. The positive commutant of a positive operator on $E$, denoted by $\{T\}_{+}^{\prime}$, is the collection of all positive operators $S$ on $E$ which commutes with $T$.

A ray $\mathcal{R}_{x_{0}}=\left\{\lambda x_{0}: \lambda \geq 0\right\}, 0 \neq x_{0} \in E_{+}$, is an extreme ray for the positive cone $E_{+}$if $x \in \mathcal{R}_{x_{0}}, x=y+z$, and $y, z \in E_{+}$imply $y, z \in \mathcal{R}_{x_{0}}$. The elements defining extreme rays are also well-known as atoms. An atom is a positive vector $x$ such that $0 \leq y \leq x$ implies $y=\lambda x$ for some $\lambda \geq 0$. A Banach lattice $E$ is called a $Q E$-space if for each pair $I, J$ of closed ideals of $E$, with $I \subseteq J$, the positive

Received by the editors December 11, 1995 and, in revised form, March 29, 1996.

1991 Mathematics Subject Classification. Primary 47B65, 47A15.

(C)1997 American Mathematical Society 
cone $(J / I)_{+}$of $J / I$ contains an extreme ray. Examples of QE-spaces are discussed in section 4. An example of a Banach lattice which is not a QE-space is given in section 5 .

An operator $T$ is said to be quasinilpotent at $x_{0}$ if $\lim _{n \rightarrow 0}\left\|T^{n} x_{0}\right\|^{1 / n}=0$. The notion of quasinilpotence at a vector was introduced first in [2], where it was shown that it plays an important role for the invariant subspace problem.

If $I$ is an ideal of $E$, then the following proposition (which we use without notice in the sequel) holds for the quotient vector space $E / I$. (See [12, Propositions II.2.6 and II.5.4].)

Proposition 1.1. For any ideal $I$ of $E, E / I$ is a vector lattice under the finest ordering of $E / I$ for which the canonical map of $E$ onto $E / I$ is positive. If $I$ is closed, then $E / I$ is a Banach lattice.

If $T \in \mathcal{B}(X)$ and if $M \in \operatorname{Lat}(T)$, then the compression of $T$ to $X / M$, denoted by $\widehat{T}$ and defined by $\widehat{T}(x+M)=T x+M$, is a well defined operator on $X / M$. In this section we state a number of lemmas concerning $T$. We omit their proofs as they are well known.

Lemma 1.2. If $T \in \mathcal{B}(E)$ is positive and if $I \in \operatorname{Ilat}(T)$, then $\widehat{T}$ is also a positive operator on $E / I$.

Lemma 1.3. Suppose $T \in \mathcal{B}(X)$ and $M \in \operatorname{Lat}(T)$.

(a) If $T$ is quasinilpotent, then $\widehat{T}$ is a quasinilpotent operator on $X / M$.

(b) If $T$ is compact, then $\widehat{T}$ is a compact operator on $X / M$.

(c) If $T$ is weakly compact, then $\widehat{T}$ is a weakly compact operator on $X / M$.

Lemma 1.4. Suppose $S, T \in \mathcal{B}(E)$ and $I \in \operatorname{Ilat}(\{S, T\})$. Let $\widehat{S}$ and $\widehat{T}$ be the compression of $S$ and $T$ to $E / I$, respectively.

(a) If $T$ is a positive operator that dominates $S$, then $\widehat{T}$ dominates $\widehat{S}$.

(b) If $S \leq T$, then $\widehat{S} \leq \widehat{T}$.

Lemma 1.5. If $I$ is a closed ideal of an $A L$-or $A M$-space $E$, then $I$ and $E / I$ are $A L$ - or AM-spaces, respectively.

Lemma 1.6. If $e$ is the unit of an AM-space $E$ and $I$ is a closed ideal of $E$, then $e+I$ is the unit of $E / I$.

\section{Decomposability theorems}

We first recall a number of known results concerning decomposability of positive operators. Here by a "positive operator" we mean "a nonzero positive operator".

Theorem 2.1 ([12, Proposition V.6.1]). If $E$ is a closed ideal of an AM-space with unit or if $E$ is a Banach lattice whose positive cone contains an extreme ray and if $T \in \mathcal{B}(E)$ is a quasinilpotent positive operator, then $T$ is decomposable.

Theorem 2.2 ([9, Proposition 2]). Every compact, quasinilpotent, positive operator on a Banach lattice is decomposable.

Theorem 2.3 ([1, Theorem 4.3]). Every compact, quasinilpotent, positive operator on a Banach lattice has a nontrivial p-hyperinvariant closed ideal.

Corollary 2.4. Suppose $T \in \mathcal{B}(E)$ is a weakly compact quasinilpotent positive operator and $E$ is an $A L$ - or $A M$-space. Then $T^{2}$ has a nontrivial p-hyperinvariant closed ideal. In particular, $T$ has a nontrivial p-hyperinvariant closed ideal. 
Proof. By [5, Corollary 5.19.9], $T^{2}$ is a compact operator. The results now follow from Theorem 2.3 and the fact that $\{T\}^{\prime} \subseteq\left\{T^{2}\right\}^{\prime}$.

Corollary 2.5. (a) Suppose $\Gamma$ is a commutative collection of positive operators on $E$ and let $T$ be a quasinilpotent positive operator. Then in each of the following cases $\Gamma$ is decomposable.

(a) $T$ is compact and $T \in \Gamma$.

(b) $T$ is dominated by a compact operator and $T^{3} \in \Gamma$.

(c) $E$ is an $A M$ - or AL-space, $T$ is weakly compact, and $T^{2} \in \Gamma$.

(d) $E$ is an $A M$ - or AL-space, $T$ is dominated by a weakly compact operator, and $T^{4} \in \Gamma$.

Proof. (a) Since $\Gamma \subseteq\{T\}_{+}^{\prime}$, Theorem 2.3 gives the desired result.

(b) By [5, Theorem 5.16.13], $T^{3}$ is compact and hence $\Gamma$ is decomposable by (a).

(c) As in the proof of Corollary 2.4, $T^{2}$ is compact. Now apply (a).

(d) By [5, Theorem 5.17.11], $T^{2}$ is weakly compact and hence $\Gamma$ is decomposable by $(\mathrm{c})$.

Our reading of [1] and [9] revealed that there is an extension of Theorem 2.3, whose proof is a slight modification of the proofs given in [9] and [1] for Theorems 2.2 and 2.3 , respectively.

Theorem 2.6. Let $T \in \mathcal{B}(E)$ be a nonzero compact positive operator. If $T$ is quasinilpotent at some $x_{0}>0$ in $E$, then $T$ has a nontrivial p-hyperinvariant closed ideal.

Proof. Since the null ideal $N_{T}=\{x \in E: T(|x|)=0\}$ is a $p$-hyperinvariant closed ideal for $T$, we are done if $T x_{0}=0$. If $T x_{0} \neq 0$ we proceed as follows:

As in the proof of [1, Theorem 4.3], $\overline{E_{u}}=F$, where

$$
F=\{x \in E: \exists y \geq 0 \text { such that }|x| \leq T y\},
$$

$u=\sum_{n=1}^{\infty} \frac{\left|x_{n}\right|}{2^{n}\left\|x_{n}\right\|}>0$, and $\left\{x_{n}\right\}$ is a norm dense sequence in $T(E)$. Hence $F$ is a nontrivial $p$-hyperinvariant closed ideal for $T$, if $E$ does not contain a quasi-interior point. Otherwise, as in the second part of [9, Proposition 2],

For each $f>0$ the closure $\overline{\mathcal{T}[f]}$ is a nonzero closed ideal which is invariant under $T$, where $\mathcal{T}[f]=\{S f: S \in \mathcal{T}\}, \mathcal{T}=\left\{S_{1}-S_{2}: S_{1}, S_{2} \in \mathcal{T}^{+}\right\}$, and $\mathcal{T}^{+}=$ $\left\{S \in \mathcal{B}(E): 0 \leq S \leq R\right.$ for some $\left.R \in\{T\}_{+}^{\prime}\right\}$.

By the last part of [1, Theorem 4.3], $\overline{\mathcal{T}[f]}$ is a $p$-hyperinvariant closed ideal for $T$; therefore we are done if we show that there exists $f \neq 0$ in $E$ such that $\overline{\mathcal{T}[f]} \neq E$. Suppose, on the contrary, that $\overline{\mathcal{T}[f]}=E$ for all $f \neq 0$, in $E$. Since $x_{0}$ and $T x_{0}$ are not equal to zero, we can choose an open ball $\mathcal{U}$, with center $x_{0}$, such that $0 \neq \overline{T(\mathcal{U})}$ and $0 \neq \overline{\mathcal{U}}$. Proceed as in the final step of the proof of [9, Proposition 2] to find a natural number $n$, a sequence $\left\{j_{m}\right\}_{m=1}^{\infty}$ in $\{1,2, \ldots, n\}$, and a sequence $\left\{S_{j}\right\}_{j=1}^{n}$ in $\mathcal{T}$ such that $g_{m}=S_{j_{m}} T S_{j_{m-1}} T \cdots S_{j_{1}} T x_{0} \in \mathcal{U}$ for all $m$, and show that $\left\|g_{m}\right\| \leq(2 C)^{m}\left\|T^{m} x_{0}\right\|$, where $C=\max \left\{\left\|R_{j}^{(i)}\right\|: j=1, \ldots, n ; i=1,2\right\}$. Since $T$ is quasinilpotent at $x_{0},\left\|g_{m}\right\| \rightarrow 0$ and hence $0 \in \overline{\mathcal{U}}$, contradicting the choice of $\mathcal{U}$.

Remark. It is clear that similar corollaries to those of Theorem 2.3 can be obtained from Theorem 2.6 if we replace quasinilpotency by quasinilpotency at some $x_{0}>0$ for which $T x_{0}>0$. 
To introduce other decomposability theorems we need to state a simple fact whose proof is omitted.

Lemma 2.7. Suppose $\Gamma \subseteq \mathcal{B}(E), T \in \mathcal{B}(E), T$ is a positive operator, and $I \in$ Ilat $(T)$. Then $I \in \operatorname{Ilat}(\Gamma)$ if either $T$ dominates all of the operators in $\Gamma$ or if all elements of $\Gamma$ are positive and $S \leq T$ for all $S \in \Gamma$.

By this Lemma and all the results preceding it, we can easily obtain other decomposability results. Here are some examples:

Corollary 2.8. Suppose $\Gamma \subseteq \mathcal{B}(E), T$ is a quasinilpotent positive operator on $E$ and either (a) $T$ dominates all members of $\Gamma$ or (b) all elements of $\Gamma$ are positive and $T$ majorizes $\Gamma$. Then in each of the following cases $\Gamma$ is decomposable; (i) $E$ is a closed ideal of an AM-space, (ii) $E$ is a Banach lattice whose positive cone contains an extreme ray, (iii) $E$ is an $A L$-space and $T$ is weakly compact, (iv) $E$ is any Banach space and $T$ is compact.

\section{IDEAL CHAINS AND IDEAL TRIANGULARIZABILITY}

The concept of triangularizability of a collection of operators on a finite or infinite dimensional Banach space has been studied by many authors, e.g., [6], [7], [8], and [10]. Recall that the collection $\Gamma$ of operators, on a Banach space, is triangularizable if there is a maximal subspace chain each of whose members is invariant under all the operators in $\Gamma$.

It is the purpose of this section to introduce a Banach lattice version of this concept and a few of its related results.

Let $A(E)$ denote the collection of all closed ideals of $E$ and partially order $A(E)$ by the inclusion relation " $\subseteq$ ". A totally ordered subset $\mathcal{F}$ of $A(E)$ will be called an ideal chain. If each element of $\mathcal{F}$ is invariant under a collection of operators $\Gamma$ on $E$, we shall call $\mathcal{F}$ an invariant ideal chain. A trivial example of an invariant ideal chain is $\{\{0\}, E\}$. The existence of nontrivial invariant ideal chains for several classes of operators on Banach lattices can be deduced from some of the theorems in section 2. A similar argument to the one given in [11, Section 4.3], shows that if $\Upsilon$, the class of all ideal chains, is partially ordered by the inclusion relation then by an application of Zorn's Lemma one can show the existence of maximal (invariant) ideal chains in $\Upsilon$.

Definition 3.1. The collection $\Gamma$ of operators on a Banach lattice $E$ is idealtriangularizable if there is a maximal ideal chain each of whose members is invariant under all the operators in $\Gamma$; such an ideal chain will be said to be triangularizing for $\Gamma$. If $\Gamma=\{T\}$, we simply say that $T$ is ideal-triangularizable.

Let $\mathcal{F}$ be an ideal chain and let $\mathcal{F}_{0}$ be a subfamily of $\mathcal{F}$. It is clear that $\Theta_{0}=$ $\bigcap\left\{I: I \in \mathcal{F}_{0}\right\}$ is a closed ideal. Since $\mathcal{F}_{0}$ is totally ordered by inclusion the set $\Omega_{0}=\bigcup\left\{I: I \in \mathcal{F}_{0}\right\}$ is a linear manifold of $E$. Now suppose $x \in \Omega_{0}$ and $y \in E$ are such that $|y| \leq|x|$. Let $I \in \mathcal{F}_{0}$ be such that $x \in I$. Since $I$ is an ideal, $y \in I$ and hence $y \in \Omega_{0}$. Thus $\Omega_{0}$ is an ideal of $E$ and hence the norm closure of $\Omega_{0}$, i.e. $\overline{\Omega_{0}}$, is a closed ideal of $E$.

Definition 3.2. Let $\mathcal{F}$ be an ideal chain. We call $\mathcal{F}$ a simple ideal chain if it satisfies: (1) $\{0\} \in \mathcal{F}$, and $E \in \mathcal{F},(2)$ if $\mathcal{F}_{0}$ is a subfamily of $\mathcal{F}$, then the closed ideals $\Theta_{0}$ and $\overline{\Omega_{0}}$ are in $\mathcal{F},(3)$ for each $J$ in $\mathcal{F}$, the quotient $J / J_{-}$is at most 
1-dimensional, where $J_{-}=[\bigcup\{I: I \in \mathcal{F}, I \neq J$, and $I \subset J\}]$ if there are closed ideals $I$ in $\mathcal{F}$ which are properly contained in $J$ and $J_{-}=\{0\}$ otherwise.

With exactly the same proof of [11, Lemma 4.3.1], one can show:

Lemma 3.3. Each simple ideal chain is maximal.

Definition 3.4. A subset $\Gamma$ of $\mathcal{B}(E)$ is said to be compressionally decomposable if for any $I, J \in \operatorname{Ilat}(\Gamma)$, with $I \subseteq J$ and $\operatorname{dim}(J / I) \geq 2$, the compression $\widehat{\Gamma}$ of $\Gamma$ to $J / I$ is decomposable.

Proposition 3.5. Suppose $E$ is a closed ideal of an AM-space $F$ with unit $f$. If $T \in \mathcal{B}(E)$ is a quasinilpotent positive operator, then $T$ is compressionally decomposable.

Proof. By Theorem 2.1 $T$ is decomposable. Let $I, J \in \operatorname{Ilat}(T)$, with $I \subseteq J$ and $\operatorname{dim}(J / I) \geq 2$. Since $E$ is an AM-space, $J$ is an AM-space. Since $I$ is a closed ideal of both $J$ and $F, J / I$ is a closed ideal of $F / I, J / I$ and $F / I$ are both AM-spaces by Lemma 1.5 , and $f+I$ is a unit for $F / I$ by Lemma 1.6.

Since $T$ is a quasinilpotent positive operator on $E, T$ is a quasinilpotent positive operator on $J$. Hence $\widehat{T}$ is a quasinilpotent positive operator on $J / I$ by Lemma 1.2 and Lemma 1.3(a). Therefore, Theorem 2.1 implies that $\widehat{T}$ is decomposable.

Proposition 3.6. Suppose $E$ is a $Q E$-space and $T$ is a quasinilpotent positive operator on $E$. Then $T$ is compressionally decomposable.

Proof. Since $E$ is a QE-space, $E_{+}=(E /\{0\})_{+}$contains an extreme ray and hence $T$ is decomposable by Theorem 2.1. Let $I, J \in \operatorname{Ilat}(T)$, with $I \subseteq J$ and $\operatorname{dim}(J / I) \geq 2$. Since $E$ is a QE-space, $(J / I)_{+}$contains an extreme ray. Since $\widehat{T}$ is a quasinilpotent positive operator on $J / I$ by Lemmas 1.2 and $1.3(\mathrm{a}), \widehat{T}$ is decomposable by Theorem 2.1.

Proposition 3.7. If $K \in \mathcal{B}(E)$ is a compact, quasinilpotent, positive operator, then $K$ and $\Gamma=\{K\}_{+}^{\prime}$ are compressionally decomposable.

Proof. By Theorem 2.3, $K$ and $\Gamma$ are decomposable. Let $I, J \in \operatorname{Ilat}(K)$ or Ilat $(\Gamma)$, with $I \subseteq J$ and $\operatorname{dim}(J / I) \geq 2$. We know that $\widehat{K}$ is a compact quasinilpotent positive operator on $J / I$ by Lemma 1.2, Lemma 1.3(a), and Lemma 1.3(b). Hence, by Theorem 2.3, $\widehat{K}$ and the compression $\widehat{\Gamma}$, of $\Gamma$ to $J / I$, are decomposable, as $\widehat{\Gamma} \subseteq\{\widehat{K}\}^{\prime}$.

Proposition 3.8. Suppose $K \in \mathcal{B}(E)$ is a weakly compact quasinilpotent positive operator and $E$ is an $A L$-or $A M$-space. Then $K$ and $\left\{K^{2}\right\}_{+}^{\prime}$ are compressionally decomposable. In particular $\{K\}_{+}^{\prime}$ is compressionally decomposable.

Proof. Apply Corollary 2.4, Lemma 1.2, Lemma 1.3 and Lemma 1.5.

Proposition 3.9. If $T \in \mathcal{B}(E)$ is a nonzero quasinilpotent positive operator and if there exists a nonzero operator $S \in\{T\}_{+}^{\prime}$ that dominates a nonzero compact operator $K \in \mathcal{B}(E)$, then $\{S, T\}$ is compressionally decomposable.

Proof. Since $T$ is a quasinilpotent positive operator, there exists $x_{0}>0$ in $E$ such that $T$ is quasinilpotent at $x_{0}$, and hence, by [3, Theorem 7.1], $\{S, T\}$ is decomposable. Suppose $I, J \in \operatorname{Ilat}(\{S, T\})$. It is easy to see that $I, J \in \operatorname{Ilat}(K)$ and, by Lemma 1.4, the compression $\widehat{S}$ of $S$ to $J / I$ dominates the compression $\widehat{K}$ 
of $K$ to $J / I$. Now apply the appropriate lemmas of section 1 and [3, Theorem 7.1] to prove $\{\widehat{S}, \widehat{T}\}$ is decomposable, if $\operatorname{dim}(J / I) \geq 2$.

The following proposition and its corollaries are easy consequences of the above results. We omit their easy proofs.

Proposition 3.10. Suppose $\Gamma \subseteq \mathcal{B}(E)$ and $T \in \Gamma$ is a compressionally decomposable positive operator. Then $\Gamma$ is compressionally decomposable if either $T$ dominates all of the elements of $\Gamma$ or if all of the elements of $\Gamma$ are positive operators and if $T$ majorizes $\Gamma$.

Corollary 3.11. Suppose $\Gamma \subseteq \mathcal{B}(E), T \in \Gamma$ is a quasinilpotent positive operator, and either (a) $T$ dominates all elements of $\Gamma$ or (b) all elements of $\Gamma$ are positive and $T$ majorizes $\Gamma$. Then $\Gamma$ is compressionally decomposable in each of the following cases: (i) $E$ is a closed ideal of an AM-space, (ii) $E$ is a $Q E$-space, (iii) $E$ is an $A L$-space and $T$ is weakly compact, (iv) $E$ is any Banach lattice and $T$ is compact.

Lemma 3.12. Suppose $\Gamma \subseteq \mathcal{B}(E)$ is compressionally decomposable. Then each maximal invariant ideal chain for $\Gamma$ is simple.

Proof. Suppose that $\mathcal{F}$ is a maximal invariant ideal chain for $\Gamma$. By a similar proof to the one given in the first part of the proof of [11, Lemma 4.3.2], we can show that $\mathcal{F}$ has the first two conditions of Definition 3.2.

To verify the third, suppose $\operatorname{dim}\left(J / J_{-}\right) \geq 2$, for some $J$ in $\mathcal{F}$. Since $\Gamma$ is compressionally decomposable, there exists a nontrivial closed ideal $I_{0}$ of $J / J_{-}$ such that $\widehat{T}\left(I_{0}\right) \subseteq I_{0}$ for all $\widehat{T} \in \widehat{\Gamma}$.

If we define $I=\left\{x \in J: x+J_{-} \in I_{0}\right\}$, then, as the canonical map $\pi: E \rightarrow E / I$ is a lattice homomorphism, $I$ is a closed ideal of $E$. It is also easy to verify that $T(I) \subseteq I$ for all $T \in \Gamma$ and $J_{-} \varsubsetneqq I \varsubsetneqq J$.

As in the last part of the proof of [11, Lemma 4.3.2], we can show that $I \notin \mathcal{F}$ and $\mathcal{F} \cup\{I\}$ is totally ordered by inclusion. Hence $\mathcal{F} \cup\{I\}$ is an invariant ideal chain for $\Gamma$ that properly contains $\mathcal{F}$, which contradicts the maximality of $\mathcal{F}$. This contradiction implies that $\operatorname{dim}\left(J / J_{-}\right)$is at most one and hence $\mathcal{F}$ is a simple ideal chain.

Theorem 3.13. Let $\Gamma \subseteq \mathcal{B}(E)$ be compressionally decomposable. Then there is a simple ideal chain $\mathcal{F}$ such that each $I \in \mathcal{F}$ is invariant under each $T \in \Gamma$.

Proof. It is sufficient to take for $\mathcal{F}$ any maximal invariant ideal chain, by Lemma 3.12 .

By using Propositions 3.5-3.10, Corollary 3.11, and Theorem 3.13, we are now able to derive some ideal-triangularizability results. We present one and leave the rest as they can be obtained similarly.

Theorem 3.14. Suppose $E$ is a closed ideal of an AM-space with unit, in particular suppose $E=C_{0}(X)$, where $X$ is a locally compact Hausdorff space. If $T$ is a quasinilpotent positive operator on $E$, then $T$ is ideal-triangularizable.

\section{CB-SPACES AND IDEAL-TRIANGULARIZABILITY}

If $X$ is a Banach space with a Schauder basis $\left\{x_{n}\right\}_{n=1}^{\infty}$, then this basis gives rise to a natural closed cone $C$ defined by $C=\left\{x=\sum_{n=1}^{\infty} \alpha_{n} x_{n}: \alpha_{n} \geq 0 \forall n\right\}$. With this cone, $X$ will be an ordered Banach space. Now if the basis is unconditional, then 
by using [13, Theorem 16.1] we can check that this ordered Banach space is indeed a Banach lattice and for every $x \in X$, with $x=\sum_{n=1}^{\infty} \alpha_{n} x_{n},|x|=\sum_{n=1}^{\infty}\left|\alpha_{n}\right| x_{n}$. Conversely, if a Banach lattice $E$ has a Schauder basis and if the positive cone of $E_{+}$ is compatible with the cone generated by this basis, then this basis is unconditional. Such Banach lattices are called $C B$-spaces.

Recently in [4], the authors established an invariant subspace theorem for the positive operators acting on a Banach space $X$ with a basis. In this section it is shown that in that theorem "subspace" can be replaced by "closed ideal" if the basis of $X$ is unconditional.

In what follows the sequence $\left\{x_{n}\right\}_{n=1}^{\infty}$ is an unconditional basis for the given CB-space.

Definition 4.1. Let $I$ be an ideal of a CB-space $E$. We say that $x_{i}$ participates in $I$ if there exists $x \in I$ with $x=\sum_{n=1}^{\infty} \alpha_{n} x_{n}$ such that $\alpha_{i} \neq 0$.

Lemma 4.2. Suppose $I$ is an ideal of a $C B$-space $E$ and $\mathcal{P}=\left\{x_{i}: x_{i}\right.$ participates in $I\}$. Then $\mathcal{P} \subset I$, and hence $I=\left\{x \in E: x=\sum_{x_{i} \in \mathcal{P}} \alpha_{i} x_{k}\right\}$.

Proof. Suppose $x_{i} \in \mathcal{P}$; then there exists $x=\sum_{n=1}^{\infty} \alpha_{n} x_{n}$ in $I$ such that $\alpha_{i} \neq 0$. Since $|x| \subset I$ and since $|x|=\sum_{n=1}^{\infty}\left|\alpha_{n}\right| x_{n}$, the relation $\left|\alpha_{i}\right| x_{i} \leq \sum_{n=1}^{\infty}\left|\alpha_{n}\right| x_{n}$ implies $\left|\alpha_{i}\right| x_{i} \in I$. Hence $x_{i} \in I$ as $\alpha_{i} \neq 0$.

Lemma 4.3. Suppose $E$ is a CB-space. Then I is a closed ideal of $E$ if and only if there exists a subset $S_{I}$ of $\mathbf{N}$ such that

$$
I=\left\{x=\sum_{n=1}^{\infty} \alpha_{n} x_{n} \in E: \alpha_{m}=0 \forall m \notin S_{I}\right\} .
$$

Proof. Suppose $I$ is a closed ideal of $E$. If $I=\{0\}$ take $S_{I}=\varnothing$, and if $I=E$ take $S_{I}=\mathbf{N}$. Suppose $I \neq\{0\}$ and $I \neq E$. If such $S_{I}$ does not exist, then for each $i \in \mathbf{N}$ there exists $x \in I$ with $x=\sum_{n=1}^{\infty} \alpha_{n} x_{n}$ such that $\alpha_{i} \neq 0$. This $x_{i}$ participates in $I$ for all $i \in \mathbf{N}$ and hence $I$ contains all elements of the basis $\left\{x_{n}\right\}_{n=1}^{\infty}$ by Lemma 4.2. Therefore, $I=E$ as $I$ is closed, a contradiction.

Conversely, suppose $S_{I}$ is a subset of $\mathbf{N}$ for which $I$ is the collection of all $x=\sum_{n=1}^{\infty} \alpha_{n} x_{n}$ in $E$ with $\alpha_{m}=0$ for all $m \notin S_{I}$. It is easy to verify that $I$ is an ideal of $E$. Now let $\left\{y_{k}\right\}_{k=1}^{\infty}$ be a sequence in $I$ that converges to $y$ in $E$. Suppose that, for each $k,\left\{\beta_{k n}\right\}_{n \in S_{I}}$ is a sequence of scalars such that $y_{k}=\sum_{n \in S_{I}} \beta_{k, n} x_{n}$. Suppose also that $\left\{\beta_{n}\right\}_{n=1}^{\infty}$ is a sequence of scalars such that $y=\sum_{n=1}^{\infty} \beta_{n} x_{n}$. Since for each $k,\left\|y_{k}-y\right\|=\left\|\sum_{n=1}^{\infty} \gamma_{k, n} x_{n}\right\|$, where $\gamma_{k, n}=\beta_{k, n}-\beta_{n}$ for $n \in S_{I}$ and $\gamma_{k, n}=-\beta_{n}$ for $n \notin S_{I}$, and since $\left\|y_{k}-y\right\| \rightarrow 0$, we should have $\lim _{k \rightarrow \infty} \beta_{k, n}=\beta_{n}$ whenever $n \in S_{I}$ and $\lim _{k \rightarrow \infty} \beta_{n}=0$ whenever $n \notin S_{I}$, as $\lim _{k \rightarrow \infty} \gamma_{k, n}=0$ for all $n \in \mathbf{N}$. Hence $\beta_{n}=0$ for all $n \notin S_{I}$, which means $y \in I$ and hence $I$ is closed.

Corollary 4.4. For each closed ideal $I \neq\{0\}$, of a $C B$-space $E$, there exists $i \in \mathbf{N}$ such that $x_{i} \in I$.

Proof. If $I=E$ there is nothing to prove. If $I \neq E$, let $S_{I}$ be as in Lemma 4.3. Since $S_{I}$ is not empty, there exists $i \in S_{I}$. For this $i$ we have $x_{i} \in I$.

Corollary 4.5. The positive cone of each closed ideal I of a CB-space E contains an extreme ray. 
Proof. If $I=\{0\}$ there is nothing to prove. Suppose $I \neq\{0\}$ and let $S_{I}$ be as in Lemma 4.3. By Corollary 4.4, let $i \in \mathbf{N}$ be such that $x_{i} \in I$. Suppose $\lambda \geq 0$ and $x, y \in I_{+}$are such that $\lambda x_{i}=x+y$, and suppose $\left\{\alpha_{n}\right\}_{n \in S_{I}},\left\{\beta_{n}\right\}_{n \in S_{I}}$ are two sequences of nonnegative scalars such that $x=\sum_{n \in S_{I}} \alpha_{n} x_{n}$ and $y=\sum_{n \in S_{I}} \beta_{n} x_{n}$. Then, by the properties of a basis we should have $\alpha_{n}+\beta_{n}=0$ for all $n \in S_{I} \backslash\{i\}$ and $\alpha_{i}+\beta_{i}=\lambda$. Hence $\alpha_{n}=\beta_{n}=0$ for all $n \in S_{I} \backslash\{i\}$. This means $x, y \in\left\{\mu x_{i}: \mu \geq 0\right\}$ and hence $I_{+}$contains an extreme ray.

Corollary 4.6. Suppose $I, J$, with $I \subseteq J$ are two closed ideals of a CB-space E. Then the positive cone $(J / I)_{+}$of the quotient space $J / I$ contains an extreme ray. Furthermore, each CB-space is a QE-space.

Proof. If either $J=\{0\}$ or $J=I$ there is nothing to prove, hence suppose $J \neq I$ and let $S_{I}$ and $S_{J}$ be as in Lemma 4.3. Clearly, $S_{i} \varsubsetneqq S_{J}$. Let $j \in S_{J}$ be such that $x_{j} \notin I$. By Corollary $4.5, x_{j}$ generates an extreme ray for $J_{+}$.

Let $x, y \in J$ be such that $x+I, y+I \in(J / I)_{+}$. (Without loss of any generality we can assume that $x, y \in J_{+}$.) Suppose the sequences of positive scalars $\left\{\alpha_{n}\right\}_{n \in S_{J}}$ and $\left\{\beta_{n}\right\}_{n \in S_{J}}$ are such that $x=\sum_{n \in S_{J}} \alpha_{n} x_{n}$ and $y=\sum_{n \in S_{J}} \beta_{n} x_{n}$. If $\lambda \geq 0$ is such that $\lambda\left(x_{j}+I\right)=(x+I)+(y+I)$, then there exists $z \in I$ such that $\lambda x_{j}-x-y=z$. Hence

$$
\left(\lambda-\alpha_{j}-\beta_{j}\right) x_{j}+\sum_{n \in S}-\left(\alpha_{n}+\beta_{n}\right) x_{n}+\sum_{n \in S_{I}}-\left(\alpha_{n}+\beta_{n}+\gamma_{n}\right) x_{n}=0,
$$

where $S=S_{J} \backslash\left(S_{I} \cup\{j\}\right)$ and $\left\{\gamma_{n}\right\}_{n \in S_{I}}$ is a sequence of scalars such that $z=$ $\sum_{n \in S_{I}} \gamma_{n} x_{n}$. Thus, $\lambda=\alpha_{j}+\beta_{j}, \alpha_{n}+\beta_{n}=0$ for all $n \in S$, and $\alpha_{n}+\beta_{n}+\gamma_{n}=0$ for all $n \in S_{I}$ and hence $\alpha_{n}=\beta_{n}=0$ for all $n \in S$. Therefore, $x+I=\alpha_{j}\left(x_{j}+I\right)$ and $y+I=\beta_{j}\left(x_{j}+I\right)$, which means $x_{j}+I$ generates an extreme ray for $(J / I)_{+}$.

Corollaries 4.6 and 3.11-(ii), and the comment preceding Theorem 3.14, now imply the main result of this section concerning CB-spaces and, in particular, the Banach lattices $c_{0}$ and $l_{p}$, where $l \leq p<\infty$. In fact:

Theorem 4.7. Suppose $E$ is a CB-space, $T$ is a quasinilpotent positive operator on $E$, and $\Gamma \subseteq \mathcal{B}(E)$. Then $T$ is ideal-triangularizable. If $T \in \Gamma$ and $T$ dominates all elements of $\Gamma$, then $\Gamma$ is also ideal-triangularizable. Furthermore, if all elements of $\Gamma$ are positive and if $T$ majorizes $\Gamma$, then $\Gamma$ is ideal-triangularizable.

\section{ExAmPles, QUeSTIONS \& COMMENTS}

Example 5.1. Let $E=L^{p}[0,1], 1 \leq p<\infty$, or $E=C[0,1]$, and let $V$ be the Volterra integral operator on $E$, i.e., the indefinite integral $V f(t)=\int_{0}^{t} f(x) d x$. It is known that $V$ is a compact quasinilpotent operator on $E$ and it is obvious that $V$ is a positive operator in both cases.

For each $s \in[0,1]$, let $I_{s}=\{f \in E: f=0$ a.e. on $[0, s]\}$ if $E=L^{p}[0,1]$, and let $I_{s}=\{f \in E: f=0$ on $[0, s]\}$ if $E=C[0,1]$. In either case it is clear that the $I_{s}$ form closed ideals of $E$ which are invariant under $V$. In a similar discussion to that of [11, Example 4.2.12], it can also be shown that, in both cases, the family $\mathcal{F}=\left\{I_{s}: 0 \leq s \leq 1\right\}$ is a simple chain, and hence $\mathcal{F}$ is a triangularizing ideal chain for $V$. 
Example 5.2. Let $K=[0,1]$ and let $E=C(K)$. For each nonzero $f \in E_{+}$, let $\alpha$ and $\beta$ be two positive numbers such that $[\alpha, \beta] \subset[0,1]$ and $f(t)>0$ for all $t \in[\alpha, \beta]$. Let $g$ be a continuous function on $[\alpha, \beta]$ such that $g(t)>0$ for all $t \in[\alpha, \beta], f(\alpha)=g(\alpha), f(\beta)=g(\beta)$, and $f(t)>g(t)$ for all $T \in(\alpha, \beta)$. Define $h$ on $[\alpha, \beta]$ by $h(t)=2 f(t)-g(t)$. Then $h$ is also continuous on $[\alpha, \beta], h(t)>0$ for all $t \in[\alpha, \beta], f(\alpha)=h(\alpha), f(\beta)=h(\beta)$, and $h(t)>f(t)$ for all $t \in(\alpha, \beta)$.

Now if we define $g, h$ on $[0, \alpha] \cup[\beta, 1]$ by $g(t)=h(t)=f(t)$, then it is easy to show that: $g, h \in E_{+}, f(t)=[g(t)+h(t)] / 2$ for all $t \in[0,1]$, and $g, h$ are not a positive multiple of $f$. This shows that $E_{+}$has no extreme rays, yet every quasinilpotent positive operator on $E$ is decomposable, by Theorem 2.1 .

Our results concerning a quasinilpotent positive operator on a CB-space give rise to the following question:

Question 5.3. Is it true that every quasinilpotent positive operator on a Banach lattice, whose positive cone contains an extreme ray, is ideal triangularizable?

Remark 5.4. The procedure in the proof of ideal-triangularizability of a quasinilpotent positive operator, on a closed ideal of an AM-space with unit or on a CBspace, may not work to answer Question 5.3. For example, the Banach lattice $E=R \oplus C[0,1]$ has an extreme ray, but, as we see in Example 5.2, the positive cone $I_{+}$of the closed ideal $I=\{0\} \oplus C[0,1]$ has no extreme rays.

In view of Theorem 7.1 of [3] and the results in [6], [7], [8], and [10] the investigation of answers to the following questions seems to be interesting.

Question 5.5. Let $T$ be a positive operator on a Banach lattice $E$ which is quasinilpotent at an element $x_{0}>0$ in $E$. Under what conditions does there exist a polynomial $p$, with positive coefficients, or $S \in\{T\}_{+}^{\prime}$ such that either $p(T)$ or $S$ dominates a nonzero compact operator on $E$ ?

Question 5.6. Under what conditions is a semigroup $\mathcal{S}$ of quasinilpotent positive operators on $E$ decomposable or ideal-triangularizable, if

(a) $E$ is a closed ideal of an AM-space or

(b) $E$ is a Banach lattice whose positive cone contains an extreme ray or

(c) $E$ is an arbitrary Banach lattice and $\mathcal{S}$ contains some compact operators?

Of course, one can obtain some partial answers, for Question 5.6, by using the results of Sections 2 and 3.

\section{ACKNOWLEDGEMENT}

This paper represents part of the author's Ph.D. research at Dalhousie University under the supervision of Heydar Radjavi.

\section{REFERENCES}

1. Y. A. Abramovich, C. D. Aliprantis and O. Burkinshaw, On the spectral radius of positive operators, Math. Z. 211 (1992), 593-607; 215 (1994), 167-168. MR 94a:47064 MR 95f:47057

2. _ Invariant subspaces of operators on $l_{p}$-spaces, J. Funct. Anal. 115 (1993), 418-424. MR 94h:47009

3. _ Invariant subspace theorems for positive operators, J. Funct. Anal. 124 (1994), 95111. MR 95e:47006

4. Invariant subspaces for positive operators acting on a Banach space with basis, Proc. Amer. Math. Soc. 123 (1995), 1773-1777. MR 95g:47005 
5. C. D. Aliprantis and O. Burkinshaw, Positive Operators, Academic Press, New York/London, 1985. MR 87h:47086

6. M.-D. Choi, E. A. Nordgren, H. Radjavi, P. Rosenthal, and Y. Zhong, Triangularizing semigroups of quasinilpotent operators with nonnegative entries, Indiana Univ. Math. J. 42 (1993), 15-25. MR 94e:47009

7. C. Laurie, E. Nordgren, H. Radjavi, and P. Rosenthal, On triangularization of algebras of operators, J. Reine. Angew. Math. 327 (1981), 143-155. MR 83d:47014

8. E. Nordgren, H. Radjavi, and P. Rosenthal, Triangularizing semigroups of compact operators, Indiana Univ. Math. J. 33 (1984), 271-275. MR 85b:47047

9. B. de Pagter, Irreducible compact operators, Math. Z. 192 (1986), 149-153. MR 87d:47052

10. H. Radjavi, On the reduction and triangularization of semigroups of compact operators, J. Operator Theory 13 (1985), 63-71. MR 86c:47056

11. R. J. Ringrose, Compact Non-self-adjoint Operators, Princeton, 1971.

12. H. H. Schaefer, Banach Lattices and Positive Operators, Springer-Verlag, Berlin/New York, 1974. MR 54:11023

13. I. Singer, Bases in Banach Spaces I, Springer-Verlag, Berlin and New York, 1970. MR 45:7451

Department of Mathematics, Dalhousie University, Halifax, Nova Scotia, Canada B3H 3J5

Current address: School of Mathematics, Isfahan University of Technology, Isfahan 84156, Iran 